\title{
Fixed Point Theorems for Monotone Mappings on Partial $D^{*}$-metric Spaces
}

\author{
N. Shobkolaei, Shaban Sedghi, S.M. Vaezpour \\ AND K.P.R. RAO*
}

\begin{abstract}
In this paper, we introduce the concept of partial $D^{*}$ metric on a nonempty set $X$. In the present paper, we give some fixed point results on these interesting spaces.
\end{abstract}

\section{INTRODUCTION}

There are a lot of fixed and common fixed point results in different type spaces. For example, metric spaces, fuzzy metric spaces and uniform spaces etc. One of the most interesting is a partial metric space, which is defined by Matthews [9]. In a partial metric space, the distance of a point to it self may not be zero. After the definition of a partial metric space, Matthews proved the partial metric version of Banach fixed point theorem. Then, Valero [21], Oltra and Valero [13] and Altun et al [3] gave some generalizations of the result of Matthews. Again, Romaguera [15] proved the Caristi type fixed point theorem on this space.

On the other hand, there have been a number of generalizations of metric spaces. One of such generalizations is a generalized metric space (or $D$ metric space) initiated by Dhage [6] in 1992. He proved the existence of unique fixed point of a self-map satisfying a contractive condition in complete and bounded $D$-metric spaces. Dealing with $D$-metric space, Ahmad et al. [1], Dhage [6, 7], Dhage et al. [8], Rhoades [14] and Singh and Sharma [20] and others made a significant contribution in fixed point theory of $D$-metric space. In 2004 Naidu et al. proved that $D$-metric is not continuous and due to this fact almost all theorems which have been proved are invalid (see $[10,11,12]$. Recently, Sh. Sedghi et al. $[16,17,18,19]$ modified the D-metric space and defined $D^{*}$-metric spaces and proved some basic properties and some fixed point and common fixed point theorems in complete $D^{*}$-metric spaces. In this paper, using the concept of $D^{*}$-metric space, we introduce

2010 Mathematics Subject Classification. Primary 54H25; Secondary 47H10.

Key words and phrases. Fixed point, partial metric.

${ }^{*}$ Corresponding author. 
the concept of partial $D^{*}$-metric space and prove a common fixed point theorem for three mappings in partial $D^{*}$-metric spaces. At first, we recall some concepts and properties of $D^{*}$-metric space.

Throughout this paper, denote $\mathbb{N}$ as the set of all natural numbers and $\mathbb{R}^{+}$as the set of all positive real numbers.

Definition 1 ([17]). Let $X$ be a nonempty set. A generalized metric (or $D^{*}$ metric) on $X$ is a function: $D^{*}: X^{3} \longrightarrow[0, \infty)$ that satisfies the following conditions for each $x, y, z, a \in X$ :

(1) $D^{*}(x, y, z) \geq 0$,

(2) $D^{*}(x, y, z)=0$ if and only if $x=y=z$,

(3) $D^{*}(x, y, z)=D^{*}(p\{x, y, z\})$,(symmetry) where $p$ is a permutation function,

(4) $D^{*}(x, y, z) \leq D^{*}(x, y, a)+D^{*}(a, z, z)$.

The pair $\left(X, D^{*}\right)$ is called a generalized metric (or $D^{*}$-metric) space.

Immediate examples of such a function are as follows.

Example 1 ([17]). (a) Let $(X, d)$ be a metric space then $D^{*}(x, y, z)=$ $\max \{d(x, y), d(y, z), d(z, x)\}$ and $D^{*}(x, y, z)=d(x, y)+d(y, z)+$ $d(z, x)$ are $D^{*}$-metric on $X$.

(b) If $X=\mathbb{R}^{n}$, then

$$
D^{*}(x, y, z)=\|x+y-2 z\|+\|y+z-2 x\|+\|z+x-2 y\|
$$

for every $x, y, z \in \mathbb{R}^{n}$ is a $D^{*}$-metric on $X$.

Example 2. Let $\psi: \mathbb{R} \times \mathbb{R} \longrightarrow \mathbb{R}^{+}$be a mapping defined as follows:

$$
\psi(x, y)=0 \text { if } x=y, \quad \psi(x, y)=\frac{1}{2} \quad \text { if } x>y, \psi(x, y)=\frac{1}{3} \text { if } x<y .
$$

Then clearly $\psi$ is not a metric, since $\psi(1,2) \neq \psi(2,1)$. Define $G: \mathbb{R} \times \mathbb{R} \times$ $\mathbb{R} \longrightarrow \mathbb{R}^{+}$by

$$
G(x, y, z)=\max \{\psi(x, y), \psi(y, z), \psi(z, x)\} .
$$

Then $G$ is a $D^{*}$-metric.

Example 3. Let $\psi: \mathbb{R}^{+} \times \mathbb{R}^{+} \longrightarrow \mathbb{R}^{+}$be a mapping defined as follows: $\psi(x, y)=\max \{x, y\}$. Then clearly it is not a metric. Define $G: \mathbb{R}^{+} \times \mathbb{R}^{+} \times$ $\mathbb{R}^{+} \longrightarrow \mathbb{R}^{+}$by

$$
G(x, y, z)=\max \{x, y\}+\max \{y, z\}+\max \{z, x\}-x-y-z,
$$

for every $x, y, z \in \mathbb{R}^{+}$. Then $G$ is a $D^{*}$-metric.

Remark 1 ([17]). In a $D^{*}$-metric space $\left(X, D^{*}\right)$, we have $D^{*}(x, x, y)=$ $D^{*}(x, y, y)$.

For more details of $D^{*}$-metric see $[16,18,19]$. 


\section{Partial $D^{*}$-Metric SPACE}

In this section we introduce the concept of a partial $D^{*}$-metric space and prove its properties.

Definition 2. A partial $D^{*}$-metric on a nonempty set $X$ is a function $p^{*}$ : $X \times X \times X \rightarrow \mathbb{R}^{+}$such that for all $x, y, z, a \in X$ :

$\left(\mathrm{p}_{1}\right) x=y=z \Longleftrightarrow p^{*}(x, x, x)=p^{*}(x, y, z)=p^{*}(y, y, y)=p^{*}(z, z, z)$,

$\left(\mathrm{p}_{2}\right) p^{*}(x, x, x) \leq p^{*}(x, y, z)$,

( $\left.\mathrm{p}_{3}\right) p^{*}(x, y, z)=p^{*}(p\{x, y, z\})$, (symmetry) where $p$ is a permutation function,

$\left(\mathrm{p}_{4}\right) p^{*}(x, y, z) \leq p^{*}(x, y, a)+p^{*}(a, z, z)-p^{*}(a, a, a)$.

A partial $D^{*}$-metric space is a pair $\left(X, p^{*}\right)$ such that $X$ is a nonempty set and $p^{*}$ is a partial $D^{*}$-metric on $X$. It is clear that, if $p^{*}(x, y, z)=0$, then from $\left(\mathrm{p}_{1}\right)$ and $\left(\mathrm{p}_{2}\right) x=y=z$. But if $x=y=z, p^{*}(x, y, z)$ may not be 0 . A basic example of a partial $D^{*}$-metric space is the pair $\left(\mathbb{R}^{+}, p^{*}\right)$, where $p^{*}(x, y, z)=\max \{x, y, z\}$ for all $x, y, z \in \mathbb{R}^{+}$.

It is easy to see that every $D^{*}$-metric is a partial $D^{*}$-metric, but the converse need not be true.

In the following examples a partial $D^{*}$-metric fails to satisfy properties of $D^{*}$-metric.

Example 4. Let $p^{*}: \mathbb{R}^{+} \times \mathbb{R}^{+} \times \mathbb{R}^{+} \longrightarrow \mathbb{R}^{+}$be a mapping defined as follows:

$$
p^{*}(x, y, z)=|x-y|+|y-z|+|x-z|+\max \{x, y, z\} .
$$

Then clearly it is a partial $D^{*}$-metric, but it is not a $D^{*}$-metric.

Example 5. Let $(X, p)$ be a partial metric space and $p^{*}: \mathbb{R}^{+} \times \mathbb{R}^{+} \times \mathbb{R}^{+} \longrightarrow$ $\mathbb{R}^{+}$be a mapping defined as follows:

$$
p^{*}(x, y, z)=p(x, y)+p(x, z)+p(y, z)-p(x, x)-p(y, y)-p(z, z) .
$$

Then clearly $p^{*}$ is a partial $D^{*}$-metric, but it is not a $D^{*}$-metric.

Remark 2. Note that $p^{*}(x, x, y)=p^{*}(x, y, y)$, because,

(i) $p^{*}(x, x, y) \leq p^{*}(x, x, x)+p^{*}(x, y, y)-p^{*}(x, x, x)=p^{*}(x, y, y)$ and similarly

(ii) $p^{*}(y, y, x) \leq p^{*}(y, y, y)+p^{*}(y, x, x)-p^{*}(y, y, y)=p^{*}(y, x, x)$.

Hence by (i) and(ii), we get $p^{*}(x, x, y)=p^{*}(x, y, y)$.

Lemma 1. Let $\left(X, p^{*}\right)$ be a partial $D^{*}$-metric space. If we define $p(x, y)=$ $p^{*}(x, y, y)$, then $(X, p)$ is a partial metric space

Proof. $\quad\left(\mathrm{p}_{1}\right) x=y \Longleftrightarrow p^{*}(x, x, x)=p^{*}(x, y, y)=p(y, y, y) \Longleftrightarrow p(x, x)=$ $p(x, y)=p(y, y)$

$\left(\mathrm{p}_{2}\right) p^{*}(x, x, x) \leq p^{*}(x, y, y)$ implies that $p(x, x) \leq p(x, y)$,

$\left(\mathrm{p}_{3}\right) p^{*}(x, y, y)=p^{*}(y, x, x)$ implies that $p(x, y)=p(y, x)$, 
$\left(\mathrm{p}_{4}\right) p^{*}(y, y, x) \leq p^{*}(y, y, z)+p^{*}(z, x, x)-p^{*}(z, z, z)$ implies that $p(x, y) \leq p(y, z)+p(z, x)-p(z, z)$.

Let $\left(X, p^{*}\right)$ be a partial $D^{*}$-metric space. For $r>0$ define

$$
B_{p^{*}}(x, r)=\left\{y \in X: p^{*}(x, y, y)<p^{*}(x, x, x)+r\right\} .
$$

Definition 3. Let $\left(X, p^{*}\right)$ be a partial $D^{*}$-metric space and $A \subset X$.

(1) If for every $x \in A$ there exists $r>0$ such that $B_{p^{*}}(x, r) \subset A$, then subset $A$ is called an open subset of $X$.

(2) A sequence $\left\{x_{n}\right\}$ in a partial $D^{*}$-metric space $\left(X, p^{*}\right)$ converges to $x$ if and only if $p^{*}(x, x, x)=\lim _{n \rightarrow \infty} p^{*}\left(x_{n}, x_{n}, x\right)$. That is for each $\varepsilon>0$ there exists $n_{0} \in \mathbb{N}$ such that

$$
p^{*}\left(x, x, x_{n}\right)<p^{*}(x, x, x)+\varepsilon \forall n \geq n_{0},
$$

or equivalently, for each $\varepsilon>0$ there exists $n_{0} \in \mathbb{N}$ such that

$$
p^{*}\left(x, x_{n}, x_{m}\right)<p^{*}(x, x, x)+\varepsilon \forall n, m \geq n_{0} .
$$

Indeed, if (1) holds then

$$
\begin{aligned}
p^{*}\left(x, x_{n}, x_{m}\right) & =p^{*}\left(x_{n}, x, x_{m}\right) \\
& \leq p^{*}\left(x_{n}, x, x\right)+p^{*}\left(x, x_{m}, x_{m}\right)-p^{*}(x, x, x) \\
& <\varepsilon+\varepsilon+p^{*}(x, x, x) .
\end{aligned}
$$

Conversely, set $m=n$ in (2) we have $p^{*}\left(x_{n}, x_{n}, x\right)<p^{*}(x, x, x)+\varepsilon$.

(3) A sequence $\left\{x_{n}\right\}$ in a partial $D^{*}$-metric space $\left(X, p^{*}\right)$ is called a Cauchy sequence if $\lim _{n, m \rightarrow \infty} p^{*}\left(x_{n}, x_{m}, x_{m}\right)$ exists.

Let $\tau_{p^{*}}$ be the set of all open subsets $X$, then $\tau_{p^{*}}$ is a topology on $X$ (induced by the partial $D^{*}$-metric $p^{*}$ ).

A partial $D^{*}$-metric space $\left(X, p^{*}\right)$ is said to be complete if every Cauchy sequence $\left\{x_{n}\right\}$ in $X$ converges, with respect to $\tau_{p^{*}}$, to a point $x \in X$.

If a sequence $\left\{x_{n}\right\}$ in a partial $D^{*}$-metric space $\left(X, p^{*}\right)$ converges to $x$ then we have

$$
\begin{aligned}
p^{*}\left(x_{n}, x_{n}, x_{m}\right) & \leq p^{*}\left(x_{n}, x_{n}, x\right)+p^{*}\left(x, x_{m}, x_{m}\right)-p^{*}(x, x, x) \\
& <\varepsilon+\varepsilon+p^{*}(x, x, x) .
\end{aligned}
$$

Lemma 2. Let $\left(X, p^{*}\right)$ be a partial $D^{*}$-metric space. If $r>0$, then ball $B_{p^{*}}(x, r)$ with center $x \in X$ and radius $r$ is an open ball. 
Proof. Let $y \in B_{p^{*}}(x, r)$, then $p^{*}(x, y, y)<p^{*}(x, x, x)+r$. Let $p^{*}(x, y, y)-$ $p^{*}(x, x, x)=\delta$. Let $z \in B_{p^{*}}(y, r-\delta)$, by triangular inequality we have

$$
\begin{aligned}
p^{*}(x, x, z) & \leq p^{*}(x, x, y)+p^{*}(y, z, z)-p^{*}(y, y, y) \\
& =p^{*}(x, y, y)-p^{*}(x, x, x)+p^{*}(z, z, y)-p^{*}(y, y, y)+p^{*}(x, x, x) \\
& <\delta+r-\delta+p^{*}(x, x, x) \\
& =p^{*}(x, x, x)+r .
\end{aligned}
$$

Thus $z \in B_{p^{*}}(x, r)$. Hence $B_{p^{*}}(y, r-\delta) \subseteq B_{p^{*}}(x, r)$. Therefore the ball $B_{p^{*}}(x, r)$ is an open ball.

Each partial $D^{*}$-metric $p^{*}$ on $X$ generates a topology $\tau_{p^{*}}$ on $X$ which has as a base the family of open $p^{*}$-balls $\left\{B_{p^{*}}(x, \varepsilon): x \in X, \varepsilon>0\right\}$.

The following example shows that a convergent sequence $\left\{x_{n}\right\}$ in a partial $D^{*}$-metric space $\left(X, p^{*}\right)$ need not be a Cauchy sequence. In particular, it shows that the limit of a convergent sequence is not necessarily unique.

Example 6. Let $X=[0, \infty)$ and $p^{*}(x, y, z)=\max \{x, y, z\}$. Then it is clear that $\left(X, p^{*}\right)$ is a complete partial $D^{*}$-metric space. Let

$$
x_{n}= \begin{cases}1, & n=2 k, \\ 2, & n=2 k+1 .\end{cases}
$$

Then clearly it is convergent sequence and for every $x \geq 2$ we have $\lim _{n \rightarrow \infty} p^{*}\left(x_{n}, x_{n}, x\right)=p^{*}(x, x, x)$, therefore

$$
L\left(x_{n}\right)=\left\{x \mid x_{n} \longrightarrow x\right\}=[2, \infty) .
$$

But $\lim _{n, m \rightarrow \infty} p^{*}\left(x_{n}, x_{m}, x_{m}\right)$ does not exist. Hence $\left\{x_{n}\right\}$ is not a Cauchy sequence.

The following lemma plays an important role in this paper.

Lemma 3. Let $(X, p)$ be a partial metric space then there exists a partial $D^{*}$-metric $p^{*}$ on $X$ such that

(a) $\left\{x_{n}\right\}$ is a Cauchy sequence in $(X, p)$ if and only if it is a Cauchy sequence in the partial $D^{*}$-metric space $\left(X, p^{*}\right)$,

(b) the partial metric space $(X, p)$ is complete if and only if the partial $D^{*}$-metric space $\left(X, p^{*}\right)$ is complete. Furthermore, $p^{*}(x, x, y)=$ $p(x, y)$ for every $x, y \in X$.

Proof. Define

$$
p^{*}(x, y, z)=\max \{p(x, y), p(x, z), p(y, z)\} \forall x, y, z \in X .
$$

Then it is easy to see that $p^{*}$ is a partial $D^{*}$-metric and $p^{*}(x, x, y)=p(x, y)$ for every $x, y \in X$.

The following Lemma shows that under certain conditions the limit is unique. 
Lemma 4. Let $\left\{x_{n}\right\}$ be a convergent sequence in a partial $D^{*}$-metric space $\left(X, p^{*}\right)$ such that $x_{n} \longrightarrow x$ and $x_{n} \longrightarrow y$. If

$$
\lim _{n \rightarrow \infty} p^{*}\left(x_{n}, x_{n}, x_{n}\right)=p^{*}(x, x, x)=p^{*}(y, y, y),
$$

then $x=y$.

Proof. As

$$
p^{*}(x, y, y)=p^{*}(x, x, y) \leq p^{*}\left(x, x, x_{n}\right)+p^{*}\left(x_{n}, y, y\right)-p^{*}\left(x_{n}, x_{n}, x_{n}\right),
$$

therefore

$$
p^{*}\left(x_{n}, x_{n}, x_{n}\right) \leq p^{*}\left(x, x, x_{n}\right)+p^{*}\left(x_{n}, y, y\right)-p(x, y, y) .
$$

By given assumptions, we have

$$
\begin{aligned}
\lim _{n \rightarrow \infty} p^{*}\left(x_{n}, x_{n}, x\right) & =p^{*}(x, x, x), \\
\lim _{n \rightarrow \infty} p^{*}\left(x_{n}, x_{n}, y\right) & =p^{*}(y, y, y), \\
\lim _{n \rightarrow \infty} p^{*}\left(x_{n}, x_{n}, x_{n}\right) & =p^{*}(x, x, x) .
\end{aligned}
$$

Therefore

$$
p^{*}(x, x, x) \leq p^{*}(x, x, x)+p^{*}(y, y, y)-p^{*}(x, y, y),
$$

which shows that $p^{*}(y, y, y) \leq p^{*}(x, y, y) \leq p^{*}(y, y, y)$. Also,

$$
p^{*}(x, y, y)=p^{*}(y, y, x) \leq p^{*}\left(y, y, x_{n}\right)+p^{*}\left(x_{n}, x, x\right)-p^{*}\left(x_{n}, x_{n}, x_{n}\right)
$$

implies that

$$
p^{*}\left(x_{n}, x_{n}, x_{n}\right) \leq p^{*}\left(y, y, x_{n}\right)+p^{*}\left(x_{n}, x, x\right)-p^{*}(x, y, y),
$$

which on taking limit as $n \rightarrow \infty$ gives

$$
p^{*}(y, y, y) \leq p^{*}(y, y, y)+p^{*}(x, x, x)-p^{*}(x, y, y),
$$

which shows that

$$
p^{*}(x, x, x) \leq p^{*}(x, y, y) \leq p^{*}(x, x, x) .
$$

Thus $p^{*}(x, x, x)=p^{*}(x, y, y)=p^{*}(y, y, y)$. Therefore $x=y$.

Lemma 5. Let $\left\{x_{n}\right\}$ and $\left\{y_{n}\right\}$ be two sequences in partial $D^{*}$-metric space $\left(X, p^{*}\right)$ such that

$$
\lim _{n \rightarrow \infty} p^{*}\left(x_{n}, x, x\right)=\lim _{n \rightarrow \infty} p^{*}\left(x_{n}, x_{n}, x_{n}\right)=p^{*}(x, x, x),
$$

and

$$
\lim _{n \rightarrow \infty} p^{*}\left(y_{n}, y, y\right)=\lim _{n \rightarrow \infty} p^{*}\left(y_{n}, y_{n}, y_{n}\right)=p^{*}(y, y, y) .
$$

Then $\lim _{n \rightarrow \infty} p^{*}\left(x_{n}, y_{n}, y_{n}\right)=p^{*}(x, y, y)$. In particular, $\lim _{n \rightarrow \infty} p^{*}\left(x_{n}, y_{n}, z\right)=$ $p^{*}(x, y, z)$ for every $z \in X$. 
Proof. As $\left\{x_{n}\right\}$ and $\left\{y_{n}\right\}$ converge to a $x \in X$ and $y \in X$ respectively, therefore for each $\varepsilon>0$ there exists $n_{0} \in \mathbb{N}$ such that

$$
\begin{aligned}
& p^{*}\left(x, x, x_{n}\right)<p^{*}(x, x, x)+\frac{\varepsilon}{2}, \\
& p^{*}\left(y, y, y_{n}\right)<p^{*}(y, y, y)+\frac{\varepsilon}{2}, \\
& p^{*}\left(x, x, x_{n}\right)<p^{*}\left(x_{n}, x_{n}, x_{n}\right)+\frac{\varepsilon}{2},
\end{aligned}
$$

and

for $n \geq n_{0}$. Now

$$
p^{*}\left(y, y, y_{n}\right)<p^{*}\left(y_{n}, y_{n}, y_{n}\right)+\frac{\varepsilon}{2}
$$

$$
\begin{aligned}
p^{*}\left(x_{n}, x_{n}, y_{n}\right) \leq & p^{*}\left(x_{n}, x_{n}, x\right)+p^{*}\left(x, y_{n}, y_{n}\right)-p^{*}(x, x, x) \\
\leq & p^{*}\left(x_{n}, x_{n}, x\right)+p^{*}\left(y, y_{n}, y_{n}\right)+p^{*}(x, x, y) \\
& \quad-p^{*}(y, y, y)-p^{*}(x, x, x) \\
< & p^{*}(x, y, y)+\frac{\varepsilon}{2}+\frac{\varepsilon}{2} \\
= & p^{*}(x, y, y)+\varepsilon,
\end{aligned}
$$

and so we have

$$
p^{*}\left(x_{n}, y_{n}, y_{n}\right)-p^{*}(x, y, y)<\varepsilon
$$

Also,

$$
\begin{aligned}
p^{*}(x, y, y) \leq & p^{*}\left(x_{n}, y, y\right)+p^{*}\left(x, x, x_{n}\right)-p^{*}\left(x_{n}, x_{n}, x_{n}\right) \\
\leq & p^{*}\left(x, x, x_{n}\right)+p^{*}\left(x_{n}, x_{n}, y_{n}\right)+p^{*}\left(y_{n}, y, y\right) \\
& \quad-p^{*}\left(y_{n}, y_{n}, y_{n}\right)-p^{*}\left(x_{n}, x_{n}, x_{n}\right) \\
< & \frac{\varepsilon}{2}+\frac{\varepsilon}{2}+p^{*}\left(x_{n}, x_{n}, y_{n}\right) \\
= & p^{*}\left(x_{n}, x_{n}, y_{n}\right)+\varepsilon .
\end{aligned}
$$

Thus

$$
p^{*}(x, x, y)-p^{*}\left(x_{n}, x_{n}, y_{n}\right)<\varepsilon .
$$

Hence for all $n \geq n_{0}$, we have $\left|p^{*}\left(x_{n}, x_{n}, y_{n}\right)-p^{*}(x, x, y)\right|<\varepsilon$. Hence the result follows.

Lemma 6. If $p^{*}$ is a partial $D^{*}$-metric on $X$, then the functions $p^{* s}, p^{* m}$ : $X \times X \times X \rightarrow \mathbb{R}^{+}$given by

$$
\begin{aligned}
p^{* S}(x, y, z) & =p^{*}(x, x, y)+p^{*}(y, y, z)+p^{*}(z, z, x) \\
& -p^{*}(x, x, x)-p^{*}(y, y, y)-p^{*}(z, z, z)
\end{aligned}
$$

and

$$
p^{* m}(x, y, z)=\max \left\{\begin{array}{l}
2 p^{*}(x, x, y)-p^{*}(x, x, x)-p^{*}(y, y, y), \\
2 p^{*}(y, y, z)-p^{*}(y, y, y)-p^{*}(z, z, z), \\
2 p^{*}(z, z, x)-p^{*}(z, z, z)-p^{*}(x, x, x)
\end{array}\right\}
$$


for every $x, y, z \in X$, are equivalent $D^{*}$-metrics on $X$.

Proof. It is easy to see that $p^{* s}$ and $p^{* m}$ are $D^{*}$-metrics on $X$. Let $x, y, z \in$ $X$. It is obvious that

$$
p^{* m}(x, y, z) \leq 2 p^{* s}(x, y, z) .
$$

On the other hand, since $a+b+c \leq 3 \max \{a, b, c\}$, it provides that

$$
\begin{aligned}
p^{* s}(x, y, z)= & p^{*}(x, x, y)+p^{*}(y, y, z)+p^{*}(z, z, x)-p^{*}(x, x, x) \\
& \quad-p^{*}(y, y, y)-p^{*}(z, z, z) \\
= & \frac{1}{2}\left[2 p^{*}(x, x, y)-p^{*}(x, x, x)-p^{*}(y, y, y)\right] \\
& \quad+\frac{1}{2}\left[2 p^{*}(y, y, z)-p^{*}(y, y, y)-p^{*}(z, z, z)\right] \\
& \quad+\frac{1}{2}\left[2 p^{*}(z, z, x)-p^{*}(z, z, z)-p^{*}(x, x, x)\right] \\
\leq & \frac{3}{2} \max \left\{\begin{array}{l}
2 p^{*}(x, x, y)-p^{*}(x, x, x)-p^{*}(y, y, y), \\
2 p^{*}(y, y, z)-p^{*}(y, y, y)-p^{*}(z, z, z), \\
2 p^{*}(z, z, x)-p^{*}(z, z, z)-p^{*}(x, x, x)
\end{array}\right\} \\
= & \frac{3}{2} p^{* m}(x, y, z) .
\end{aligned}
$$

Thus,we have

$$
\frac{1}{2} p^{* m}(x, y, z) \leq p^{* s}(x, y, z) \leq \frac{3}{2} p^{* m}(x, y, z) .
$$

These inequalities implies that $p^{* s}$ and $p^{* m}$ are equivalent.

Remark 3. Note that:

$$
p^{* S}(x, x, y)=2 p^{*}(x, x, y)-p^{*}(x, x, x)-p^{*}(y, y, y)=p^{* m}(x, x, y) .
$$

A mapping $F: X \rightarrow X$ is said to be continuous at $x_{0} \in X$, if for every $\varepsilon>0$, there exists $\delta>0$ such that $F\left(B_{p^{*}}\left(x_{0}, \delta\right)\right) \subseteq B_{p^{*}}\left(F x_{0}, \varepsilon\right)$.

The following lemma plays an important role to prove fixed point results on a partial $D^{*}$-metric space.

Lemma 7. Let $\left(X, p^{*}\right)$ be a partial $D^{*}$-metric space.

(a) $\left\{x_{n}\right\}$ is a Cauchy sequence in $\left(X, p^{*}\right)$ if and only if it is a Cauchy sequence in the $D^{*}$-metric space $\left(X, p^{* s}\right)$.

(b) A partial $D^{*}$-metric space $\left(X, p^{*}\right)$ is complete if and only if the $D^{*}$ metric space $\left(X, p^{* S}\right)$ is complete. Furthermore,

$$
\lim _{n \rightarrow \infty} p^{* s}\left(x_{n}, x_{n}, x\right)=0
$$

if and only if

$$
p^{*}(x, x, x)=\lim _{n \rightarrow \infty} p^{*}\left(x_{n}, x_{n}, x\right)=\lim _{n, m \rightarrow \infty} p^{*}\left(x_{n}, x_{n}, x_{m}\right) .
$$


Proof. First we show that every Cauchy sequence in $\left(X, p^{*}\right)$ is a Cauchy sequence in $\left(X, p^{* s}\right)$. To this end let $\left\{x_{n}\right\}$ be a Cauchy sequence in $\left(X, p^{*}\right)$. Then there exists $\alpha \in \mathbb{R}$ such that, for given $\varepsilon>0$, there is $n_{\varepsilon} \in \mathbb{N}$ with $\left|p^{*}\left(x_{n}, x_{n}, x_{m}\right)-\alpha\right|<\frac{\varepsilon}{4}$ for all $n, m \geq n_{\varepsilon}$. Hence

$$
\begin{aligned}
p^{* s}\left(x_{n}, x_{n}, x_{m}\right)= & \mid 2 p^{*}\left(x_{n}, x_{n}, x_{m}\right)-p^{*}\left(x_{n}, x_{n}, x_{n}\right) \\
& \quad-p^{*}\left(x_{m}, x_{m}, x_{m}\right)+2 \alpha-2 \alpha \mid \\
\leq & \left|2 p^{*}\left(x_{n}, x_{n}, x_{m}\right)-2 \alpha\right|+\left|p^{*}\left(x_{n}, x_{n}, x_{n}\right)-\alpha\right| \\
& \quad+\left|p^{*}\left(x_{m}, x_{m}, x_{m}\right)-\alpha\right| \\
< & 4 \frac{\varepsilon}{4}=\varepsilon,
\end{aligned}
$$

for all $n, m \geq n_{\varepsilon}$. Which implies that $\left\{x_{n}\right\}$ is a Cauchy sequence in $\left(X, p^{* s}\right)$. Next we prove that completeness of $\left(X, p^{* S}\right)$ implies completeness of $\left(X, p^{*}\right)$. Indeed, if $\left\{x_{n}\right\}$ is a Cauchy sequence in $\left(X, p^{*}\right)$ then it is also a Cauchy sequence in $\left(X, p^{* s}\right)$. Since the $D^{*}$-metric space $\left(X, p^{* S}\right)$ is complete we deduce that there exists $y \in X$ such that $\lim _{n \rightarrow \infty} p^{* s}\left(x_{n}, x_{n}, y\right)=0$. Therefore,

$$
\begin{aligned}
& \limsup _{n \rightarrow \infty}\left|p^{*}\left(x_{n}, x_{n}, y\right)-p^{*}(y, y, y)\right| \\
& \leq \lim _{n \rightarrow \infty}\left|2 p^{*}\left(x_{n}, x_{n}, y\right)-p^{*}\left(x_{n}, x_{n}, x_{n}\right)-p^{*}(y, y, y)\right|=0 .
\end{aligned}
$$

Hence we follow that $\left\{x_{n}\right\}$ is a convergent sequence in $\left(X, p^{*}\right)$. That is,

$$
\lim _{n \rightarrow \infty} p^{*}\left(x_{n}, x_{n}, y\right)=p^{*}(y, y, y) .
$$

Now we prove that every Cauchy sequence $\left\{x_{n}\right\}$ in $\left(X, p^{* s}\right)$ is a Cauchy sequence in $\left(X, p^{*}\right)$. Let $\varepsilon=\frac{1}{2}$, then there exists $n_{0} \in \mathbb{N}$ such that $p^{* s}\left(x_{n}, x_{n}, x_{m}\right)<$ $\frac{1}{2}$ for all $n, m \geq n_{0}$. Since

$$
\begin{aligned}
p^{*}\left(x_{n}, x_{n}, x_{n}\right) \leq & 4 p^{*}\left(x_{n_{0}}, x_{n_{0}}, x_{n}\right)-3 p^{*}\left(x_{n}, x_{n}, x_{n}\right) \\
& \quad-p^{*}\left(x_{n_{0}}, x_{n_{0}}, x_{n_{0}}\right)+p^{*}\left(x_{n}, x_{n}, x_{n}\right) \\
\leq & 2 p^{* s}\left(x_{n}, x_{n}, x_{n_{0}}\right)+p^{*}\left(x_{n_{0}}, x_{n_{0}}, x_{n_{0}}\right) .
\end{aligned}
$$

Thus, we have

$$
\begin{aligned}
p^{*}\left(x_{n}, x_{n}, x_{n}\right) & \leq 2 p^{* s}\left(x_{n}, x_{n}, x_{n_{0}}\right)+p^{*}\left(x_{n_{0}}, x_{n_{0}}, x_{n_{0}}\right) \\
& \leq 1+p^{*}\left(x_{n_{0}}, x_{n_{0}}, x_{n_{0}}\right) .
\end{aligned}
$$

Consequently the sequence $\left\{p^{*}\left(x_{n}, x_{n}, x_{n}\right)\right\}$ is bounded in $\mathbb{R}$, and so there exists an $a \in \mathbb{R}$ such that a subsequence $\left\{p^{*}\left(x_{n_{k}}, x_{n_{k}}, x_{n_{k}}\right)\right\}$ is convergent to $a$, i.e. $\lim _{k \rightarrow \infty} p^{*}\left(x_{n_{k}}, x_{n_{k}}, x_{n_{k}}\right)=a$.

It remains to prove that $\left\{p^{*}\left(x_{n}, x_{n}, x_{n}\right)\right\}$ is a Cauchy sequence in $\mathbb{R}$. Since $\left\{x_{n}\right\}$ is a Cauchy sequence in $\left(X, p^{* s}\right)$, for given $\varepsilon>0$, there exists $n_{\varepsilon}$ such 
that $p^{* s}\left(x_{n}, x_{n}, x_{m}\right)<\frac{\varepsilon}{2}$ for all $n, m \geq n_{\varepsilon}$. Thus, for all $n, m \geq n_{\varepsilon}$,

$$
\begin{aligned}
\left|p^{*}\left(x_{n}, x_{n}, x_{n}\right)-p^{*}\left(x_{m}, x_{m}, x_{m}\right)\right| \leq & 4 p^{*}\left(x_{n}, x_{n}, x_{m}\right) \\
& -3 p^{*}\left(x_{n}, x_{n}, x_{n}\right)-p^{*}\left(x_{m}, x_{m}, x_{m}\right) \\
& +p^{*}\left(x_{n}, x_{n}, x_{n}\right)-p^{*}\left(x_{m}, x_{m}, x_{m}\right) \\
\leq & 2 p^{* s}\left(x_{n}, x_{n}, x_{m}\right)<\varepsilon .
\end{aligned}
$$

On the other hand,

$$
\begin{aligned}
\left|p^{*}\left(x_{n}, x_{n}, x_{n}\right)-a\right| \leq & \left|p^{*}\left(x_{n}, x_{n}, x_{n}\right)-p^{*}\left(x_{n_{k}}, x_{n_{k}}, x_{n_{k}}\right)\right| \\
& +\left|p^{*}\left(x_{n_{k}}, x_{n_{k}}, x_{n_{k}}\right)-a\right| \\
<\varepsilon & +\varepsilon=2 \varepsilon,
\end{aligned}
$$

for all $n, n_{k} \geq n_{\varepsilon}$. Hence $\lim _{n \rightarrow \infty} p^{*}\left(x_{n}, x_{n}, x_{n}\right)=a$.

Now, we show that $\left\{x_{n}\right\}$ is a Cauchy sequence in $\left(X, p^{*}\right)$. We have,

$$
\begin{aligned}
&\left|2 p^{*}\left(x_{n}, x_{n}, x_{m}\right)-2 a\right|= \mid p^{* s}\left(x_{n}, x_{n}, x_{m}\right)+p^{*}\left(x_{n}, x_{n}, x_{n}\right) \\
&-a+p^{*}\left(x_{m}, x_{m}, x_{m}\right)-a \mid \\
& \leq p^{* s}\left(x_{n}, x_{n}, x_{m}\right)+\left|p^{*}\left(x_{n}, x_{n}, x_{n}\right)-a\right| \\
& \quad+\left|p^{*}\left(x_{m}, x_{m}, x_{m}\right)-a\right| \\
&<\frac{\varepsilon}{2}+2 \varepsilon+2 \varepsilon=\frac{9}{2} \varepsilon .
\end{aligned}
$$

That is, $\left\{x_{n}\right\}$ is a Cauchy sequence in $\left(X, p^{*}\right)$.

We shall have established the lemma if we prove that $\left(X, p^{* s}\right)$ is complete if so is $\left(X, p^{*}\right)$. Let $\left\{x_{n}\right\}$ be a Cauchy sequence in $\left(X, p^{* S}\right)$. Then $\left\{x_{n}\right\}$ is a Cauchy sequence in $\left(X, p^{*}\right)$, and so it is convergent to a point $y \in X$ with,

$$
\lim _{n, m \rightarrow \infty} p^{*}\left(x_{n}, x_{n}, x_{m}\right)=\lim _{n \rightarrow \infty} p^{*}\left(y, y, x_{n}\right)=p^{*}(y, y, y) .
$$

Thus, for given $\varepsilon>0$, there exists $n_{\varepsilon} \in \mathbb{N}$ such that

$$
p^{*}\left(y, y, x_{n}\right)-p^{*}(y, y, y)<\frac{\varepsilon}{2} \text { and }\left|p^{*}(y, y, y)-p^{*}\left(x_{n}, x_{n}, x_{n}\right)\right|<\frac{\varepsilon}{2}
$$

whenever $n \geq n_{\varepsilon}$. As a consequence we have

$$
\begin{aligned}
p^{* s}\left(y, y, x_{n}\right) & =2 p^{*}\left(y, y, x_{n}\right)-p^{*}\left(x_{n}, x_{n}, x_{n}\right)-p^{*}(y, y, y) \\
& \leq\left|p^{*}\left(y, y, x_{n}\right)-p^{*}(y, y, y)\right|+\left|p^{*}\left(y, y, x_{n}\right)-p^{*}\left(x_{n}, x_{n}, x_{n}\right)\right| \\
& <\frac{\varepsilon}{2}+\frac{\varepsilon}{2}=\varepsilon,
\end{aligned}
$$

whenever $n \geq n_{\varepsilon}$. Therefore $\left(X, p^{* s}\right)$ is complete.

Finally, it is a simple matter to check that $\lim _{n \rightarrow \infty} p^{* s}\left(a, a, x_{n}\right)=0$ if and only if

$$
p^{*}(a, a, a)=\lim _{n \rightarrow \infty} p^{*}\left(a, a, x_{n}\right)=\lim _{n, m \rightarrow \infty} p^{*}\left(x_{n}, x_{n}, x_{m}\right) .
$$


Definition 4. Let $\left(X, p^{*}\right)$ be a partial $D^{*}$-metric space, then $p^{*}$ is said to be of the first type if for every $x, y \in X$ we have

$$
p^{*}(x, x, y) \leq p^{*}(x, y, z),
$$

for every $z \in X$.

\section{Fixed point Result}

We begin this section giving the concept of weakly increasing mappings (see [5]).

Definition 5. Let $(X, \preceq)$ be a partially ordered set. Two mappings $S, T$ : $X \longrightarrow X$ are said to be $S-T$ weakly increasing if $S x \preceq T S x$ for all $x \in X$.

Note that, two weakly increasing mappings need not be nondecreasing. There exist some examples to illustrate this fact in [4].

In the sequel, we use the following notations:

(i) $\mathcal{F}$ denote the set of all functions $F:[0, \infty) \longrightarrow[0, \infty)$ such that $F$ is nondecreasing and continuous, $F(0)=0<F(t)$ for every $t>0$ and $F(x+y) \leq F(x)+F(y)$ for all $x, y \in[0,+\infty)$;

(ii) $\Psi$ denote the set of all functions $\psi:[0, \infty) \longrightarrow[0, \infty)$ where $\psi$ is continuous, nondecreasing function such that $\sum_{n=0}^{\infty} \psi^{n}(t)$ is convergent for each $t>0$. From the conditions on $\psi$, it is clear that $\lim _{n \rightarrow \infty} \psi^{n}(t)=0$ and $\psi(t)<t$ for every $t>0$.

Our main result is as follows:

Theorem 1. Let $(X, \preceq)$ be a partially ordered set and suppose that there exists a first type partial $D^{*}$-metric $p^{*}$ on $X$ such that $\left(X, p^{*}\right)$ is a complete partial $D^{*}$-metric space.

Let $S, T, R: X \longrightarrow X$ are three $S-T, T-R$ and $R-S$ weakly increasing mappings such that

$$
F\left(p^{*}(S x, T y, R z)\right) \leq \psi(F(\varphi(x, y, z)))
$$

for all $x, y, z \in X$ with $x, y, z$ are comparable with respect to partially order $\preceq$, where $F \in \mathcal{F}, \psi \in \Psi$ and

$$
\varphi(x, y, z)=\max \left\{\begin{array}{l}
p^{*}(x, y, z), p^{*}(x, x, S x), \\
p^{*}(y, y, T y), p^{*}(z, z, R z)
\end{array}\right\} .
$$

Further assume that if for every increasing sequence $\left\{x_{n}\right\}$ convergent to $x \in$ $X$ we have $x_{n} \preceq x$.

Then $S, T$ and $R$ have a common fixed point.

Proof. Let $x_{0}$ be an arbitrary point of $X$. We can define a sequence in $X$ as follows:

$x_{3 n+1}=S x_{3 n}, x_{3 n+2}=T x_{3 n+1} \quad$ and $\quad x_{3 n+3}=R x_{3 n+2} \quad$ for $\quad n=0,1, \ldots$ 
Since $S, T, R$ are three $S-T, T-R$ and $R-S$ weakly increasing mappings, we have

$$
x_{1}=S x_{0} \preceq T S x_{0}=x_{2}=T x_{1} \preceq R T x_{1}=x_{3}=R x_{2} \preceq S R x_{2}=x_{4}
$$

and continuing this process we have

$$
x_{1} \preceq x_{2} \cdots \preceq x_{n} \preceq x_{n+1} \preceq \cdots .
$$

Case: Suppose there exists $n_{0} \in \mathbb{N}$ such that $p^{*}\left(x_{3 n_{0}}, x_{3 n_{0}+1}, x_{3 n_{0}+2}\right)=0$. Now we show that $p^{*}\left(x_{3 n_{0}+1}, x_{3 n_{0}+2}, x_{3 n_{0}+3}\right)=0$. Otherwise, from (3.1), we get

$$
\begin{aligned}
F\left(p^{*}\left(x_{3 n_{0}+2}, x_{3 n_{0}+2}, x_{3 n_{0}+3}\right)\right) & \leq F\left(p^{*}\left(x_{3 n_{0}+1}, x_{3 n_{0}+2}, x_{3 n_{0}+3}\right)\right) \\
& =F\left(p^{*}\left(S x_{3 n_{0}}, T x_{3 n_{0}+1}, R x_{3 n_{0}+2}\right)\right) \\
& \leq \psi\left(F\left(\varphi\left(x_{3 n_{0}}, x_{3 n_{0}+1}, x_{3 n_{0}+2}\right)\right)\right) \\
& =\psi\left(F\left(x_{3 n_{0}+2}, x_{3 n_{0}+2}, x_{3 n_{0}+3}\right)\right) \\
& \left.<F\left(x_{3 n_{0}+2}, x_{3 n_{0}+2}, x_{3 n_{0}+3}\right)\right),
\end{aligned}
$$

which is a contradiction. Hence $p^{*}\left(x_{3 n_{0}+1}, x_{3 n_{0}+2}, x_{3 n_{0}+3}\right)=0$. Therefore, $x_{3 n_{0}}=x_{3 n_{0}+1}=x_{3 n_{0}+2}=x_{3 n_{0}+3}$. Thus $S x_{3 n_{0}}=T x_{3 n_{0}}=R x_{3 n_{0}}=x_{3 n_{0}}$. That is $x_{3 n_{0}}$ is a common fixed point of $S, T$ and $R$.

Case: Assume that $p^{*}\left(x_{3 n}, x_{3 n+1}, x_{3 n+2}\right)>0$ for every $n \in \mathbb{N}$. Now we prove that

$$
F\left(p^{*}\left(x_{n-1}, x_{n}, x_{n+1}\right)\right) \leq \psi\left(F\left(p^{*}\left(x_{n-2}, x_{n-1}, x_{n}\right)\right)\right) .
$$

Setting $x=x_{3 n}, y=x_{3 n+1}$ and $z=x_{3 n+2}$ in (3.2), we have

$$
\varphi\left(x_{3 n}, x_{3 n+1}, x_{3 n+2}\right)=\max \left\{\begin{array}{l}
p^{*}\left(x_{3 n}, x_{3 n+1}, x_{3 n+2}\right), \\
p^{*}\left(x_{3 n}, x_{3 n}, x_{3 n+1}\right), \\
p^{*}\left(x_{3 n+1}, x_{3 n+1}, x_{3 n+2}\right), \\
p^{*}\left(x_{3 n+2}, x_{3 n+2}, x_{3 n+3}\right)
\end{array}\right\} .
$$

Since, $p^{*}$ is of the first type, we get $\varphi\left(x_{3 n}, x_{3 n+1}, x_{3 n+2}\right) \leq \max \left\{p^{*}\left(x_{3 n}, x_{3 n+1}, x_{3 n+2}\right), p^{*}\left(x_{3 n+1}, x_{3 n+2}, x_{3 n+3}\right)\right\}$. If $p^{*}\left(x_{3 n+1}, x_{3 n+2}, x_{3 n+3}\right)$ is maximum in the R.H.S. of the above inequality, we have from (3.1)that

$$
\begin{aligned}
F\left(p^{*}\left(x_{3 n+1}, x_{3 n+2}, x_{3 n+3}\right)\right) & =F\left(p^{*}\left(S x_{3 n}, T x_{3 n+1}, R x_{3 n+2}\right)\right) \\
& <\psi\left(F\left(\varphi\left(x_{3 n}, x_{3 n+1}, x_{3 n+2}\right)\right)\right) \\
& \leq \psi\left(F \left(\operatorname { m a x } \left\{p^{*}\left(x_{3 n}, x_{3 n+1}, x_{3 n+2}\right),\right.\right.\right. \\
& \left.\left.\left.\quad p^{*}\left(x_{3 n+1}, x_{3 n+2}, x_{3 n+3}\right)\right\}\right)\right) \\
& =\psi\left(F\left(p^{*}\left(x_{3 n+1}, x_{3 n+2}, x_{3 n+3}\right)\right)\right) \\
& <F\left(p^{*}\left(x_{3 n+1}, x_{3 n+2}, x_{3 n+3}\right)\right),
\end{aligned}
$$


which is a contradiction. Thus,

$$
F\left(p^{*}\left(x_{3 n+1}, x_{3 n+2}, x_{3 n+3}\right)\right) \leq \psi\left(F\left(p^{*}\left(x_{3 n}, x_{3 n+1}, x_{3 n+2}\right)\right) .\right.
$$

Similarly, we have

$$
F\left(p^{*}\left(x_{3 n+2}, x_{3 n+3}, x_{3 n+4}\right)\right) \leq \psi\left(F\left(p^{*}\left(x_{3 n+1}, x_{3 n+2}, x_{3 n+3}\right)\right)\right),
$$

and

$$
F\left(p^{*}\left(x_{3 n}, x_{3 n+1}, x_{3 n+2}\right)\right) \leq \psi\left(F\left(p^{*}\left(x_{3 n-1}, x_{3 n}, x_{3 n+1}\right)\right)\right) .
$$

Therefore, for every $n \in \mathbb{N}$ we have

$$
F\left(p^{*}\left(x_{n}, x_{n+1}, x_{n+2}\right)\right) \leq \psi\left(F\left(p^{*}\left(x_{n-1}, x_{n}, x_{n+1}\right)\right)\right) .
$$

Now, we have

$$
F\left(p^{*}\left(x_{n}, x_{n+1}, x_{n+2}\right)\right) \leq \psi\left(F\left(p^{*}\left(x_{n-1}, x_{n}, x_{n+1}\right)\right)\right) \leq \cdots \leq \psi^{n}\left(F\left(p^{*}\left(x_{0}, x_{1}, x_{2}\right)\right)\right) .
$$

Hence

$$
\lim _{n \rightarrow \infty} F\left(p^{*}\left(x_{n}, x_{n+1}, x_{n+2}\right)\right)=0,
$$

so that

$$
\lim _{n \rightarrow \infty} p^{*}\left(x_{n}, x_{n+1}, x_{n+2}\right)=0 .
$$

Since $p^{*}$ is of the first type and $F$ is nondecreasing, we have

$$
F\left(p^{*}\left(x_{n}, x_{n}, x_{n+1}\right) \leq F\left(p^{*}\left(x_{n}, x_{n+1}, x_{n+2}\right)\right) \leq \psi^{n}\left(F\left(p^{*}\left(x_{0}, x_{1}, x_{2}\right)\right)\right) .\right.
$$

Since $F(x+y) \leq F(x)+F(y)$ and $p^{* s}\left(x_{n}, x_{n}, x_{n+1}\right) \leq 2 p^{*}\left(x_{n}, x_{n}, x_{n+1}\right)$ we have

$$
F\left(p^{* s}\left(x_{n}, x_{n}, x_{n+1}\right) \leq 2 F\left(p^{*}\left(x_{n}, x_{n}, x_{n+1}\right)\right) \leq 2 \psi^{n}\left(F\left(p^{*}\left(x_{0}, x_{1}, x_{2}\right)\right)\right) .\right.
$$

Now from $p^{* s}\left(x_{n+k}, x_{n}, x_{n}\right) \leq p^{* s}\left(x_{n+k}, x_{n+k-1}, x_{n+k-1}\right)+\cdots+p^{* s}\left(x_{n+1}, x_{n}, x_{n}\right)$, we have

$$
\begin{aligned}
F\left(p^{* s}\left(x_{n+k}, x_{n}, x_{n}\right)\right) & \leq F\left(p^{* s}\left(x_{n+k}, x_{n+k-1}, x_{n+k-1}\right)\right)+\cdots+F\left(p^{* s}\left(x_{n+1}, x_{n}, x_{n}\right)\right) \\
& \leq 2 \psi^{n+k-1}\left(p^{*}\left(x_{0}, x_{1}, x_{2}\right)\right)+\cdots+2 \psi^{n}\left(p^{*}\left(x_{0}, x_{1}, x_{2}\right)\right) \\
& \leq 2 \sum_{i=n}^{\infty} \psi^{i}\left(p^{*}\left(x_{0}, x_{1}, x_{2}\right)\right) .
\end{aligned}
$$

Since $\sum_{n=1}^{\infty} \psi^{n}(t)$ is convergent for each $t>0$ it follows that $\left\{x_{n}\right\}$ is a Cauchy sequence in the $D^{*}$-metric space $\left(X, p^{* s}\right)$. Since $\left(X, p^{*}\right)$ is complete, then from Lemma 7 follows that the sequence $\left\{x_{n}\right\}$ converges to some $x$ in the $D^{*}$-metric space $\left(X, p^{* s}\right)$. Hence $\lim _{n \rightarrow \infty} p^{* s}\left(x_{n}, x, x\right)=0$. Again, from Lemma 7 , we have

$$
p^{*}(x, x, x)=\lim _{n \rightarrow \infty} p^{*}\left(x_{n}, x, x\right)=\lim _{n, m \rightarrow \infty} p^{*}\left(x_{n}, x_{m}, x_{m}\right) .
$$

Since $\left\{x_{n}\right\}$ is a Cauchy sequence in the $D^{*}$-metric space $\left(X, p^{* S}\right)$ and

$$
p^{* s}\left(x_{n}, x_{m}, x_{m}\right)=2 p^{*}\left(x_{n}, x_{m}, x_{m}\right)-p^{*}\left(x_{n}, x_{n}, x_{n}\right)-p^{*}\left(x_{m}, x_{m}, x_{m}\right),
$$


we have

$$
\lim _{n, m \rightarrow \infty} p^{* s}\left(x_{n}, x_{m}, x_{m}\right)=0
$$

and by (3.4) we have

$$
\lim _{n \rightarrow \infty} p^{*}\left(x_{n}, x_{n}, x_{n}\right)=0
$$

thus by definition $p^{* s}$ we have

$$
\lim _{n, m \rightarrow \infty} p^{*}\left(x_{n}, x_{m}, x_{m}\right)=0 .
$$

Therefore by (3.5), we have

$$
\begin{aligned}
p^{*}(x, x, x) & =\lim _{n \rightarrow \infty} p^{*}\left(x_{n}, x, x\right) \\
& =\lim _{n, m \rightarrow \infty} p^{*}\left(x_{n}, x_{m}, x_{m}\right)=0 .
\end{aligned}
$$

Now by the inequality (3.1) for $x=x, y=x_{3 n+1}$ and $z=x_{3 n+2}$, then we have

$$
F\left(p^{*}\left(S x, x_{3 n+2}, x_{3 n+3}\right)\right) \leq \psi\left(F\left(\varphi\left(x, x_{3 n+1}, x_{3 n+2}\right)\right),\right.
$$

and by letting $n \rightarrow \infty$ and using Lemma 5 , we obtain

$$
F\left(p^{*}(S x, x, x)\right) \leq \psi\left(F\left(p^{*}(S x, x, x)\right)<F\left(p^{*}(S x, x, x)\right),\right.
$$

which is a contradiction. Hence, $p^{*}(S x, x, x)=0$. Thus $S x=x$. Similarly, by using the inequality (3.1) for $y=x, x=x_{3 n}$ and $z=x_{3 n+2}$, then we have

$$
F\left(p^{*}\left(x_{3 n}, T x, x_{3 n+3}\right)\right) \leq \psi\left(F\left(\varphi\left(x_{3 n}, x, x_{3 n+2}\right)\right),\right.
$$

and letting $n \rightarrow \infty$ and using Lemma 5, we obtain

$$
F\left(p^{*}(x, T x, x)\right) \leq \psi\left(F\left(p^{*}(x, T x, x)\right)<F\left(p^{*}(x, T x, x)\right),\right.
$$

which is a contradiction.

Hence, $p^{*}(x, T x, x)=0$. Thus $T x=x$. Similarly, by using the inequality (3.1) for $z=x, x=x_{3 n}$ and $y=x_{3 n+1}$, we can show that $R x=x$.

Corollary 1. Let $(X, \preceq)$ be a partially ordered set and suppose that there exists a first type partial $D^{*}$-metric $p^{*}$ on $X$ such that $\left(X, p^{*}\right)$ is a complete partial $D^{*}$-metric space.

Let $S: X \longrightarrow X$ be a mapping such that $S x \preceq S^{2} x$ and

$$
F\left(p^{*}(S x, S y, S z)\right) \leq \psi(F(\varphi(x, y, z)))
$$

for all $x, y, z \in X$ with $x, y, z$ are comparable with respect to partially order $\preceq$, where $F \in \mathcal{F}, \psi \in \Psi$ and

$$
\varphi(x, y, z)=\max \left\{\begin{array}{l}
p^{*}(x, y, z), p^{*}(x, x, S x), \\
p^{*}(y, y, S y), p^{*}(z, z, S z) .
\end{array}\right\}
$$

Further assume that if for every increasing sequence $\left\{x_{n}\right\}$ convergent to $x \in$ $X$ we have $x_{n} \preceq x$.

Then $S$ has a fixed point. 
Example 7. Let $X=[0, \infty)$ and $p^{*}(x, y, z)=\max \{x, y, z\}$, then $\left(X, p^{*}\right)$ is a partial $D^{*}$-metric space.

Define self-map $S$ on $X$ as $S x=\frac{x}{2}$, and the relation $\preceq$ on $X$ as follows:

$$
x \preceq y \Longleftrightarrow x \geq y,
$$

for any $x, y \in X$. Then $\preceq$ is a (partial) order on $X$ induced by $\leq$. Since $S x \geq S^{2} x$ it follows that $S x \preceq S^{2} x$. If define $F(t)=t$ and $\psi(t)=k t$ for $0<k<1$ then it is easy to see that

$$
p^{*}(S x, S y, S z) \leq k \varphi(x, y, z),
$$

for every $x$ in $X$ and $\frac{1}{2} \leq k<1$. Thus all conditions of Corollary 1 are satisfied and $x=0$ is the unique fixed point of $S$.

\section{REFERENCES}

[1] B. Ahmad, M. Ashraf and B.E. Rhoades, Fixed point theorems for expansive mappings in D-metric spaces, Indian J. Pure Appl. Math., 32 (2001), 1513-1518.

[2] I. Altun and A. Erduran, Fixed point theorems for monotone mappings on partial metric spaces, Fixed Point Theory Appl., Art. ID 508730 (2011), 10 pages.

[3] I. Altun, F. Sola and H. Simsek, Generalized contractions on partial metric spaces, Topology and its Applications, 157 (2010), 2778-2785.

[4] I. Altun and H. Simsek, Some fixed point theorems on ordered metric spaces and application, Fixed Point Theory and Appl., Article ID 621492 (2010), 17 pages.

[5] I. Beg and A.R. Butt, Fixed point for set-valued mappings satisfying an implicit relation in partially ordered metric spaces, Nonlinear Anal., 71 (2009), 3699-3704.

[6] B.C. Dhage, Generalized metric spaces and mappings with fixed point, Bull. Calcutta Math. Soc., 84 (1992), no. 4, 329-336.

[7] B.C. Dhage, A common fixed point principe in D-metric spaces, Bull. Calcutta Math. Soc., 91 (1999), 475-480.

[8] B.C. Dhage, A.M. Pathan and B.E. Rhoades, A general existence priciple for fixed point theorem in D-metric spaces, Int. J. Math. Math. Sci., 23 (2000), 441-448.

[9] S.G. Matthews, Partial metric topology, Proc. 8th Summer Conference on General Topology and Applications, Ann. New York Acad. Sci., 728 (1994), 183-197.

[10] S.V.R. Naidu, K.P.R. Rao and N. Srinivasa Rao, On the topology of D-metric spaces and the generation of D-metric spaces from metric spaces, Internat. J. Math. Math. Sci., 2004 (2004), No. 51, 2719-2740.

[11] S.V.R. Naidu, K.P.R. Rao and N. Srinivasa Rao, On the concepts of balls in a Dmetric space, Internat. J. Math. Math. Sci., 2005, No. 1 (2005), 133-141.

[12] S.V.R. Naidu, K.P.R. Rao and N. Srinivasa Rao, On convergent sequences and fixed point theorems in D-metric spaces, Internat. J. Math. Math. Sci., 2005:12 (2005), 1969-1988.

[13] S. Oltra and S. Valero, Banach's fixed point theorem for partial metric spaces, Rend. Istid. Math. Univ. Trieste, 36 (2004), 17-26.

[14] B.E. Rhoades, A fixed point theorem for generalized metric spaces, Int. J. Math. Math. Sci., 19 (1996), no. 1, 145-153.

[15] S. Romaguera, A Kirk type characterization of completeness for partial metric spaces, Fixed Point Theory and Applications, Article ID 493298 (2010), 6 pages. 
[16] Sh. Sedghi, K.P.R. Rao and N. Shobe, Common fixed point theorems for six weakly compatible mappings in $D^{*}$-metric spaces, International Journal of Mathematical Sciences, Vol. 6, No. 2 (2007), 225-237.

[17] Sh. Sedghi, N. Shobe and H. Zhou, A common fixed point theorem in $D^{*}$-metric spaces, Fixed point Theory and Applications, Article ID 27906 (2007), 13 pages.

[18] Sh. Sedghi, D. Turkoglu, N. Shobe and S. Sedghi, Common Fixed Point Theorems for Six Weakly Compatible Mappings in $D^{*}$-Metric Spaces, Thai Journal of Mathematics, 7 (2009) Number 2, 381-391.

[19] Sh. Sedghi, N. Shobe and S. Sedghi, Common fixed point theorems for two mappings in $D^{*}$-metric spaces, Journal of Prime Research in Mathematics, Vol. 4 (2008), 132142.

[20] B. Singh and R.K. Sharma, Common fixed points via compatible maps in D-metric spaces, Rad. Mat., Vol. 11 (2002), no. 1, 145-153.

[21] O. Valero, On Banach fixed point theorems for partial metric spaces, Appl. General Topology, Vol. 6 (2005), 229-240.

\author{
N. Shobkolaei \\ Department of Mathematics, \\ SCIEnce And Research Branch \\ ISLAMIC AZAD UNIVERSITY \\ 1477893855 TEHRAN \\ IRAN \\ E-mail address: nabi_shobe@yahoo.com
}

\section{Shaban Sedghi}

Department of Mathematics

QAEMSHAHR BRANCH

ISLAMiC AZAD UNIVERSITY

QAEMSHAHR

IRAN

E-mail address: sedghi_gh@yahoo.com

sedghi.gh@qaemshahriau.ac.ir

\section{S.M. VAEzPour}

Department of Mathematics and Computer Science Amirkabir University of Technology

424 Hafez Avenue

TEHRAN 15914

IRAN

E-mail address: vaez@aut.ac.ir

\section{K.P.R. RAO}

Department of Mathematics

ACharya Nagarjuna University

NAGARJUNA NAGAR-522 510

Guntur District, Andhra Pradesh

INDIA

E-mail address: kprrao2004@yahoo.com 